\title{
Acceleration from Modified Gravity: Lessons from Worked Examples
}

\author{
Wayne $\mathrm{Hu}^{\mathrm{a} *}$

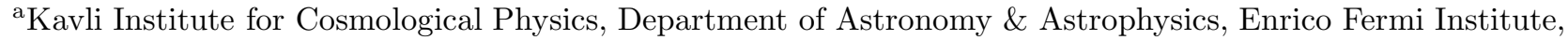 \\ University of Chicago, Chicago, IL 60637
}

I examine how two specific examples of modified gravity explanations of cosmic acceleration help us understand some general problems confronting cosmological tests of gravity: how do we distinguish modified gravity from dark energy if they can be made formally equivalent? how do we parameterize deviations according to physical principles with sufficient generality, yet focus cosmological tests into areas that complement our existing knowledge of gravity? how do we treat the dynamics of modifications which necessarily involve non-linearities that preclude superposition of forces? The modified action $f(R)$ and DGP braneworld models provide insight on these question as fully-worked examples whose expansion history, linear perturbation theory, and most recently, non-linear $N$-body and force-modification field dynamics of cosmological simulations are available for study.

\section{INTRODUCTION}

The next generation of surveys aimed at characterizing the acceleration of the expansion will also provide precision tests of gravity in a cosmological context. In particular, they will address the possibility that the acceleration itself is driven not by an unknown dark energy component but rather by a modification to gravity on the largest scales (e.g. [1/2/3/4]).

In the absence of a compelling theory for modified gravity, how do we best quantify and focus these tests? Is there an analogue of the parameterized postNewtonian description of local gravity for cosmology? Can it act as a meeting point between observations and theory?

At the very outset there are some basic questions that confront this endeavor. In particular, three issues, loosely based on the challenges compiled in 4, appear as obstacles:

1. Dark energy equivalence. Formally, any metric theory of modified gravity can be recast into an equivalent dark energy theory under ordinary gravity. Is a cosmological test of gravity itself even possible?

2. Anything goes. Without the framework of general relativity we are left with arbitrary functions of space and time to relate the matter and the metric. What guidance do we have to parameterize them?

\footnotetext{
*This work was supported by the KICP through grants NSF PHY0114422 and NSF PHY-0551142, U.S. Dept. of Energy contract DE-FG02-90ER-40560 and the David and Lucile Packard Foundation. I thank W. Fang, M. Lima, L. Lombriser, H. Oyaizu, I. Sawicki, F. Schmidt, YS. Song, A. Upadhye for fruitful collaborations; L. Hui, J. Khoury, K. Koyama, B. Wald for illuminating discussions; GGI and the organizers of the New Horizons workshop for their hospitality.
}

3. Superposition. Order unity cosmological deviations and tiny local deviations imply that the modified Poisson equation is non-linear in some way. How do we treat non-linear dynamics without a superposition principle for gravitational forces?

Given such basic issues, it is useful to have concrete examples, however contrived, ad hoc, or problematic on a quantum level. They serve as case studies for how a modified gravity theory might work.

Two such examples are the modified action model where the Einstein-Hilbert action is supplemented by a non-linear function of the Ricci scalar $f(R) 55$, 7] and the 5-dimensional braneworld model of Dvali, Gabadadze, \& Porrati (DGP) 8]. Both cases are now fully worked in that their expansion history, linear perturbation theory, and most recently, non-linear $N$-body dynamics of cosmological simulations have been studied.

In this piece, I examine how these worked examples provide insight on the three conundrums confronting cosmological tests of gravity for cosmic acceleration.

\section{DARK ENERGY EQUIVALENCE}

Given the formal equivalence which maps a modified gravity theory onto a dark energy theory under ordinary gravity, can cosmological observables ever test gravity itself?

Let's examine the formal equivalence and then place it in the context of the two worked examples. A metric theory of gravity where energy-momentum is covariantly (locally) conserved $\left(\nabla^{\mu} T_{\mu \nu}=0\right)$ can always be rewritten as a dark energy theory. To see this, consider that a generally covariant field equation of the form (e.g. 9[10])

$S_{\mu \nu}\left(g_{\mu \nu}\right)=8 \pi G T_{\mu \nu}$ 
can be re-expressed as ordinary gravity $G_{\mu \nu}=8 \pi G T_{\mu \nu}$ with an effective dark energy stress energy

$T_{\mu \nu}^{\mathrm{DE}} \equiv \frac{1}{8 \pi G}\left(G_{\mu \nu}-S_{\mu \nu}\right)$.

Moreover this effective stress-energy is covariantly conserved $\nabla^{\mu} T_{\mu \nu}^{\mathrm{DE}}=0$ by virtue of the Bianchi identity. Also, conservation of the matter means that the only influence of the modification to gravity or effective dark energy comes through the metric: once the gravitational potentials are determined, matter falls in them as usual.

While this formal equivalence holds, it does not mean that the equivalent dark energy description obeys reasonable microphysics. Microphysics determine the equations of state that relate the components of the stress energy tensor: the pressure, energy density, momentum density and anisotropic stress. The effective dark energy description of a modified gravity model will look contrived as its microphysics must mimic an explicit dependence on the metric unlike in physical dark energy models like scalar fields [10].

Moreover, even though the matter and effective dark energy are separately conserved, the relationship between the effective microphysics and the metric imply an implicit coupling between the dark energy and matter - i.e. the effective dark energy mediates a fifth forces that universally couples to the matter. The $f(R)$ and DGP models are both exhibit this phenomenology. They contain an extra scalar degree of freedom that can either be represented as dark energy anisotropic stress, proportional to the metric, or the propagating mediator of a fifth force. As such, they are phenomenologically quite distinct from typical dark energy models.

In summary, cosmological tests of gravity, just like solar system measurements, require a notion of what composes the stress-energy tensor(s) in the system. For cosmological tests, we assume candidates for the dark matter and dark energy with microphysics that, along with conservation of energy-momentum, closes their equations of motion [11. What most cosmologists implicitly mean by a "test of gravity" is: a test of gravity in the context of cold dark matter and dark energy whose density is relatively spatially smooth on scales well below the current horizon 2 In this context, general relativity makes specific and falsifiable predictions for cosmological observables. Falsification however does not necessarily imply that general relativity is incorrect.

\section{ANYTHING GOES}

Without the framework of general relativity how do we parameterize and test gravity cosmologically?

${ }^{2}$ Modified gravity explanations of the dark matter fall into a different category and are not the subject of this exploration.
Consistency Approaches: If anything goes, then shouldn't one start with parameters that define the simplest self-consistency tests of general relativity (plus dark energy that is smooth relative to cold dark matter)?

Candidates for self-consistency tests include an approximate expression for the growth rate of linear density perturbations $\Delta_{m}$ given the matter contribution to the expansion rate $\Omega_{m}(a)$ (e.g. [12[13])

$\frac{d \ln \Delta_{m}}{d \ln a} \approx \Omega_{m}(a)^{\gamma}$,

where $\gamma \approx 0.55$. Other approaches include separate dark energy equation of states that govern growth and distance measures (e.g. 14[15]) or model independent reconstructions of growth from distance measures that can be compared with growth measurements (e.g. [16[17]). The latter have the advantage that consistency checks can be made exact in linear theory whereas the former two parametric schemes are easier to apply.

One problem with these approaches is that deviations in these parameters away from consistency do not represent physically possible modified gravity models. As minimal approaches, they also do not target areas where measurements might best complement what we already know about gravity. For example, consistency statistics can be dominated by information from the non-linear regime where, as we shall see, ordinary gravity is almost guaranteed. Conversely, an incomplete account of non-linear baryonic physics can give false consistency violations [18.

Likewise, characteristic signatures of modified gravity aren't very well exposed by these consistency statistics. Neither the $f(R)$ nor DGP models have a scale independent linear growth rate, though deviations for the latter only appear near the horizon scale [19]20|21]. Moreover modified gravity theories such as these change the relationship between the gravitational potential, spatial curvature and the density perturbations.

It is tempting to simply supplement consistency parameters like $\gamma$, generalized perhaps to be a function of scale, with a variation in the Newton constant $G$ that also depends on scale (e.g. 222). However these types of prescriptions do not establish a framework where physical principles like conservation of energy-momentum strictly apply nor do they provide a viable construction beyond linear perturbations.

Despite these flaws, simple consistency tests are valuable in that they are easy to apply and forecast. Their violation will point to new physics or astrophysics that can then be more fully developed. They are not however the only reasonable metric to apply for judging tests of gravity.

Linear Parameterization: Fortunately, the problem of 
formal equivalence to dark energy in $\ 2$ and knowledge that gravity must only have small deviations from general relativity locally (see (4) is a virtue for developing a parameterized description of modified gravity.

The framework of general relativity and aspects of the parameterized post Newtonian description for deviations carry over to modified gravity. Coupled with specific worked examples of the types of deviations expected in modified gravity models, this framework brings form to a cosmological parameterization of gravity.

As discussed in $\$ 2$, if we can parameterize the degrees of freedom associated with the microphysics of the effective dark energy then we have a description that satisfies physical principles: gravity as a metric theory and and conservation of energy-momentum.

Modified gravity theories, including the two worked examples, typically have an extra scalar degree of freedom associated with the physics that drives the acceleration. Just as in the parameterized post-Newtonian (PPN) description, this scalar characterizes the relationship between the Newtonian potential $\Psi$ and the spatial curvature $\Phi$ but now in the context of small deviations from a statistically homogeneous and isotropic Friedmann-Robertson-Walker background

$$
\begin{aligned}
g & =\frac{\Phi+\Psi}{\Phi-\Psi} \\
d s^{2} & =-(1+2 \Psi) d t^{2}+a^{2}(1+2 \Phi) d x^{2}
\end{aligned}
$$

Note that $g=0$ for a universe composed of cold dark matter and smooth dark energy. These deviations can be measured by comparing gravitational lensing and gravitational redshifts, which are sensitive to $\Phi-\Psi$, with the dynamics of non-relativistic matter, which is sensitive to $\Psi$.

In terms of the effective dark energy, the metric deviation $g$ is related to anisotropic stress (e.g. [23/24]). On superhorizon scales, this parameter $g(a, k \rightarrow 0)$ alone is sufficient to describe the evolution of the two potentials $\Phi, \Psi$ given a known, separately parameterized, expansion history 25]. Completeness follows from the conservation of the comoving curvature for adiabatic perturbations

$\zeta=$ const. $\quad(k \ll a H)$

independently of the microphysics of dark energy. This independence can also be directly shown without the aid of the dark energy equivalence [26]. Fundamentally, it only requires that conservation of energy-momentum still apply.

This fact is also quite useful for describing the two worked modified gravity examples. In the DGP scenario, the complicated superhorizon physics of metric fluctuations propagating into the extra dimension through the master equation for the bulk is simply encapsulated by this one function on the brane [27. The inclusion of this horizon scale effect leads to an excess in the large angle CMB anisotropy [28] which brings the self-accelerating solution into $5 \sigma$ tension with the joint CMB and supernovae data 29]. Likewise, the complicated 4th order dynamics of $f(R)$ theories is also neatly encapsulated in $g$ on large scales [19].

On smaller scales, conservation of energy-momentum and $g$ is not sufficient for a complete description. Again dark energy equivalence is useful for understanding this fact. There it is necessary to close the equations of motion by supplementing conservation with an equation of state that relates the pressure fluctuation to the density, momentum and anisotropic stress fluctuations. For a smooth dark energy component, this is achieved by setting a Jeans scale or a sound speed for pressure and anisotropic stress fluctuations for the dark energy [11.

One possibility that has been explored [24]30] is setting the anisotropic stress and pressure of the effective dark energy to exactly cancel so as to leave no source to the momentum density in a particular gauge choice, synchronous gauge. This amounts to assuming that $\zeta$ is a constant on all scales if the remaining matter is cold dark matter and corresponds to a case where the effective dark energy has non-negligible density contributions on small scales 3 However it is too restrictive and does not lead to a small scale description that has the features of the worked examples.

In both the DGP and viable $f(R)$ models, the Newtonian regime is well approximated by an unmodified Poisson equation for the lensing potential

$\nabla^{2}\left(\frac{\Phi-\Psi}{2}\right)=-4 \pi G a^{2} \bar{\rho}_{m} \Delta_{m}$

where spatial derivatives are comoving throughout. Along with $g$ and energy-momentum conservation for the matter, the Poisson equation for the lensing potential completes the system. More generally $G$ can be made time dependent without breaking the structure of these equations [10. On the other hand, parameterizations that just involve $g$ and the Poisson equation [31|22|32] fail to automatically enforce energymomentum conservation or the Bianchi identity on horizon scales and above. For the $f(R)$ model $g \rightarrow-1 / 3$ below the Compton wavelength scale in the background and for the DGP model $g \rightarrow-1 / 3 \beta(a)$ where $\beta(a)=$ $1-2 H r_{c}\left(1+\frac{1}{3} d \ln H / d \ln a\right)$ with $r_{c}$ as the crossover scale.

\footnotetext{
${ }^{3}$ The same issue applies to synchronous gauge techniques that simply shut off dark energy perturbations on small scales as an approximation to smooth dark energy: in a synchronous gauge choice where the dark matter defines the frame, the momentum density of the dark energy is never negligible.
} 
A parameterized post-Friedmann (PPF) description that is flexible enough to incorporate both the large and small scale behavior of linear fluctuations in the two worked examples for modified gravity was introduced in [10. The gist of this parameterization is to include an interpolation between the large scale Friedmann behavior of equation (6) and the Newtonian behavior of equation (77) while strictly conserving energy-momentum on all scales. This amounts to highly non-trivial closure relations or equations of state for the equivalent effective dark energy. Such complexity addresses the question of distinguishability raised in $\$ 2$,

These relations are readily generalized to the early universe where radiation fields from the photons and neutrinos are important and provide their own anisotropic stress [33. A public code to calculate linear fluctuations including the CMB under this generalized PPF description was released in [34. 4 This method has also been adapted to handle smooth dark energy, braneworld gravity with brane tension [35, and explorations of degravitation ideas [36.

Non-linear Parameterization: There is one final, perhaps most critical, piece to a PPF description. Given that there exists very strong constraints on analogous PPN parameters locally, e.g. in solar system tests, there must be some means by which finite modifications at cosmological scales are reduced to acceptable levels locally.

Here is where having the worked examples prove the most valuable. In the DGP and $f(R)$ scenarios, ordinary gravity is restored by having non-linear interactions of the force-modification field in the presence of non-linear matter fluctuations. It is not sufficient to simply have $g(a, k)$ run with wavenumber since the restoration works in real space and is dependent on the local environment. The PPF approach in [10] for 2-point statistics takes the rms density field as an interpolation parameter between linearized modified gravity and non-linear ordinary gravity inside collapsed dark matter halos. This approach works quite well for DGP where the non-linearity is linked closely with density [37. The original PPF approach requires generalization for other non-linear mechanisms which interpolate between the linear and deeply non-linear regime in different ways and for statistics beyond 2-point functions

While the non-linear PPF description is still incomplete, it is crucial that a parameterized scheme incorporate at least one degree of freedom which allows gravity to return to normal locally. Without this feature one might infer overly tight limits on modifications to gravity in the linear regime from non-linear statistics such as the cluster abundance or cosmic shear (cf. 38]). This

$\sqrt[4]{\text { camb.info/ppf }}$ caveat applies to consistency parameters like $\gamma$ as well. We turn now to the guidance the non-linear dynamics of the worked examples provide on these and related issues.

\section{SUPERPOSITION}

Given that the return to ordinary gravity locally necessitates some non-linear process, the superposition principle for forces among bodies no longer applies. How then do we test gravity with cosmological observables in the non-linear regime? Does the lack of a superposition principle, which implies mode coupling, invalidate even large-scale predictions based on linear theory?

Non-linear Field Dynamics: The most critical and difficult piece of a viable description for modified gravity is how non-linear effects return ordinary gravity locally. The two worked examples $f(R)$ and DGP provide some guidance on how this can happen. In both cases, on small scales and assuming non-relativistic velocities for the matter, Eq. 7 for the lensing potential continues to apply but the non-linear equivalent to $g$ comes from a modified Poisson equation

$\nabla^{2} \Psi=4 \pi G a^{2} \bar{\rho}_{m} \Delta_{m}+\frac{1}{2} \nabla^{2} \phi$,

where the extra scalar $\phi=-d f / d R$ in $f(R)$ theories and is associated with the brane position in DGP. Note that the Poisson equation is still linear in the density fluctuation from the mean $\Delta_{m}$ but gains an extra source from a scalar degree of freedom $\nabla^{2} \phi$.

Given that the matter still moves in the metric as usual, non-relativistic particles still feel forces according to $\nabla \Psi$. Nonetheless compared with ordinary gravity there is an enhanced gravitational (or "fifth") force from $\nabla \phi$. This extra source is set in linear theory to be $\phi=$ $\Phi+\Psi=g(\Phi-\Psi)$ and so should obey the equation

$\nabla^{2} \phi=g(a) a^{2}\left(8 \pi G \bar{\rho}_{m} \Delta_{m}\right)$, (linear, $\left.k \gg a H\right)$.

To suppress the enhanced forces locally, the non-linear generalization should have the form

$\nabla^{2} \phi=g(a) a^{2}\left(8 \pi G \bar{\rho}_{m} \Delta_{m}-N[\phi]\right)$,

where $g(a)$ is now taken to be the small scale limit of the linear relationship between the metric potentials. Here $N[\phi]$ is some non-linear function of the field and its derivatives which goes to

$N[\phi] \rightarrow 8 \pi G \bar{\rho}_{m} \Delta_{m}$

locally. In the $f(R)$ case, $N[\phi]=\delta R(f(\phi))$ and is a function of the local field alone. Here, the general relativistic expectation that $R=8 \pi G \rho_{m}$ gives the minimum of the effective potential for $\phi$. If the minimum can be reached inside an overdense region then force law deviations are 
shielded in the interior by the so-called chameleon mechanism (e.g. 39).

In the DGP case

$N[\phi]=\frac{r_{c}^{2}}{a^{4}}\left[\left(\nabla^{2} \phi\right)^{2}-\left(\nabla_{i} \nabla_{j} \phi\right)^{2}\right]$.

Here the non-linearity contains quadratic combinations of second derivatives of the field just like the Laplace operator on the lhs of Eq. (10). Detailed balance suggests that once $\nabla^{2} \phi$ becomes large, the non-linear piece will be driven to cancel $8 \pi G \bar{\rho}_{m} \Delta_{m}$ with the field being a nearly algebraic function of the overdensity. In this case, the suppression is called the Vainshtein mechanism.

$N$-body Techniques: The generic features of the nonlinear dynamics from the worked examples are: the Poisson equation for $\Psi$ remains linear in its sources of the density fluctuation and the Laplacian of the forcemodification field with the matter falling in the metric as usual. Once $\Psi$ is obtained other aspects of mesh-based $N$-body simulations remains the same.

On the other hand, the scalar source, like the density field, obeys a non-linear equation of motion and its configuration in the presence of the density field must be solved numerically. The non-linear field equation can be solved with iterative relaxation techniques [40]. These have been successfully applied to the $f(R)$ [4] and DGP sources of non-linearity [42] in a particle-mesh N-body. Inside a large overdensity, the scalar source responds non-linearly and suppresses the additional contribution to gravitational forces. Non-linearity implies that the gravitational field is no longer the sum of all of the independent contributions from individual bodies, instead there is a collective saturation of the sources of deviation from ordinary gravity.

This saturation makes scaling relations between linear theory predictions and non-linear observables established for ordinary gravity dangerous to apply to modified gravity. These include relationships between the linear growth rate and the non-linear power spectrum and halo mass function 41/43/42.

It is not even guaranteed that the average scalar source follows the linear theory prediction on large supposedly linear scales. Lack of a superposition principle breaks the linear assumption of averaging: small scale non-linear density fluctuations can affect the behavior of the large-scale field. In a universe where even large scale density fluctuations are composed from the correlations between non-linear collapsed dark matter halos, this feature can invalidate the predictions of linear theory. Likewise, the non-linear contributions to the scalar field around a collapsed body can alter the field contribution to the metric substantially. As a consequence it can no longer be thought of as moving as a test body in an external field. We must solve self-consistently for the internal and external field.

Superposition and Saturation: There are important similarities and differences between the ways $f(R)$ and DGP resolve the superposition and saturation issues. In both cases, $N$-body results show that large scale cosmological density fluctuations do obey linear dynamics despite the lack of a superposition principle.

In the $f(R)$ case, saturation of the scalar source occurs as the field itself saturates in value inside an overdense region, $\phi \rightarrow 0$. More specifically, field saturation occurs when the gravitational potential of an object exceeds the value of the background field $\bar{\phi}$.

A thought example where all of the matter resides in objects with such deep gravitational potentials exposes some counterintuitive properties of the lack of superposition. Since the field contribution is suppressed then in all regions containing the matter, their contribution to the total gravitational potential in Eq. (8) reflects ordinary gravity. Thus the large scale gravitational potential will not follow the predictions of linear theory with modified gravity. Likewise, the motion of these collapsed objects would also not follow the large-scale external gradients of the field (set say by the clustering of voids). The saturation of the field internal to the object would eliminate the influence of any external gradient for particles within the object (see Fig. 10).

This effect can be phrased as an apparent equivalence principle violation between saturated and unsaturated objects as pointed out by [44. That a saturated object requires a non-linear field response means that by definition saturated objects considered as a whole are not test particles. Note that the matter that composes these objects still are test particles and so these still respond to the total metric including the non-superimposable internal and external field contributions. As such, there is no fundamental equivalence principle violation since matter, regardless of its composition, will always fall on geodesics of the total, local metric.

In the cosmological $f(R)$ simulations for cases with force law deviations in the linear regime, the dark matter halos where most of the matter resides do not possess deep enough gravitational potentials to be fully saturated. In such cases, the linear theory predictions are valid and apparent equivalence principle violations between dark matter halos of different mass are difficult to identify.

In the DGP case, the non-linearity involves second derivatives of the field. Let us first consider the case where the the $\nabla_{i} \nabla_{j} \phi$ and $\nabla^{2} \phi$ terms are comparable, and for simplicity proportional [45],

$N[\phi]=\frac{r_{c}^{2}}{a^{4}} s\left(\nabla^{2} \phi\right)^{2}$. 


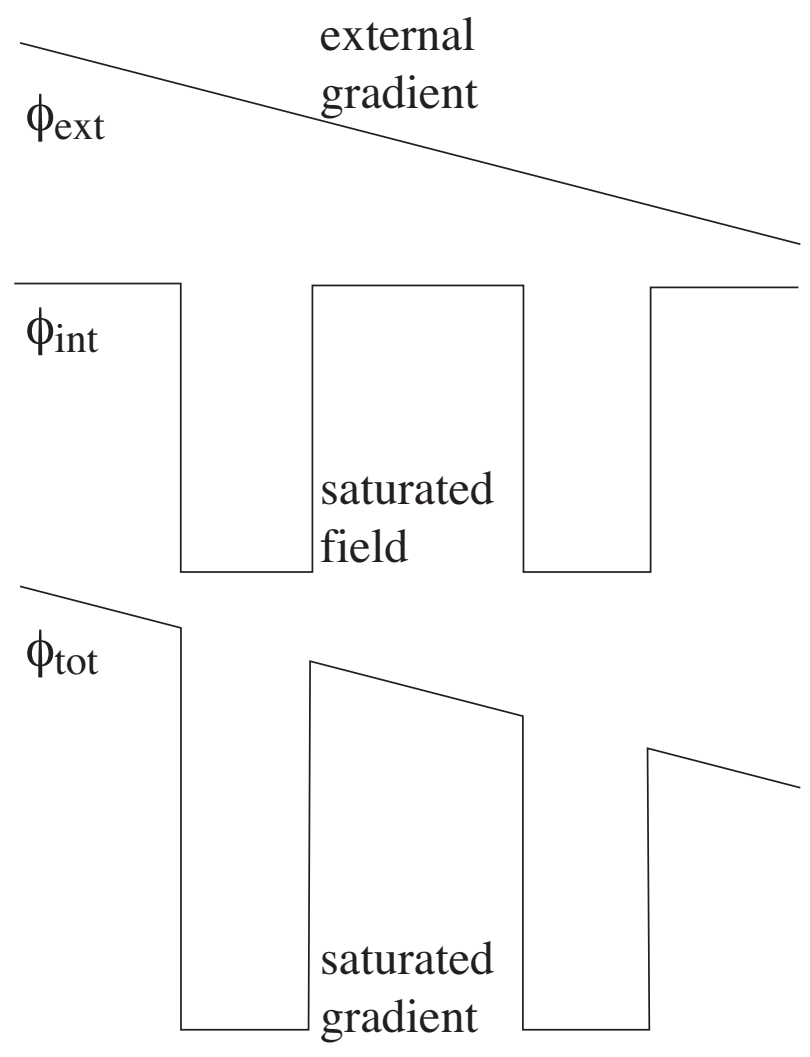

Figure 1. Non-linear saturation of field gradients in $f(R)$ (exaggerated for effect). For a screened object, the internal field $\phi_{\text {int }}$ generated by the object does not superimpose with an external field $\phi_{\text {ext }}$. In particular, the field inside the object loses knowledge of any exterior gradient and the enhanced gravitational force it implies. Screened objects are by definition not test bodies with respect to the field but still respond gravitationally to the total field $\phi_{\text {tot }}$ and hence the metric.

For example, the interior field of an isolated spherically symmetric tophat density configuration would have $\phi=$ $c_{1} r^{2}+c_{2}$ and hence $s=2 / 3$ [6]45].

The non-linear field equation then becomes an algebraic relationship between the scalar source and the density with the solution [46]

$\nabla^{2} \phi \approx \frac{a^{2}}{2 g r_{c}^{2} s}\left[\sqrt{1+32 \pi G g^{2} r_{c}^{2} s \bar{\rho}_{m} \Delta_{m}}-1\right]$.

At low density $\nabla^{2} \phi=g(a) a^{2}\left(8 \pi G \bar{\rho}_{m} \Delta_{m}\right)$ as desired whereas at high density the source is suppressed $\nabla^{2} \phi \propto$ $\Delta_{m}^{1 / 2}$. Given the DGP form for $g$, the threshold for the change in behavior scales as $\Delta_{m} \approx 1 / s$ and so the suppression of the field source is determined by the nonlinearity of the local density contrast [4]. Contrast this with $f(R)$ where the chameleon transition is linked to the local gravitational potential.
In the modified Poisson equation (8), this looks like a density dependent Newton constant. If this were the whole story, DGP would exhibit much stronger violations of superposition than $f(R)$. Since much of the total mass of the universe is in small collapsed objects with $\Delta_{m}>1$, these sources would contribute less to the large scale modifications to gravity than their average would imply as in our thought example above. This is problematic for $N$-body simulations based on this approximation [45]. Even the large scale ("linear") predictions depend on the resolution: as more and more of the mass is resolved into collapsed objects the modification to forces on large scales will be reduced.

Fortunately, this is not the case for the full DGP nonlinear term. Returning to the isolated source case, the field configuration far away from the source (beyond the Vainshtein radius) reveals an unscreened source due to the $\nabla_{i} \nabla_{j} \phi$ terms [45]44]. Hence as long as the linear regime scales are larger than the Vainshtein radius of the collapsed objects, the lack of a superposition principle does not violate linear theory predictions. This fact is also related to the absence of an apparent equivalence principle violation in DGP 44.

Finally, it is interesting to note that for a planar configuration $N[\phi]=0$ for DGP and there is no non-linear suppression even when $\Delta_{m} \gg 1$. This has the interesting consequence that cosmological structure that is planar or sheetlike exhibits the largest modification to gravity. These counterterms that suppress the non-linearity for planar configurations are a generic feature of models with "galileon" shift symmetry [48.

\section{DISCUSSION}

Perhaps the primary lesson of the worked examples is that gravity is fragile and preserving what we know about gravity already poses severe restrictions on a viable modification that explains cosmic acceleration. On the other hand, this fragility provides form to parametric approaches and insight on how to design cosmological tests that best complement our knowledge of gravity.

Demanding a metric theory where the matter obeys a conservation law requires that deviations take the form of a dark energy component under ordinary gravity. The most important parameter for modified gravity in this language is an effective dark energy anisotropic stress that is coupled directly to the lensing potential. The resulting phenomenology is therefore distinct from typical physical dark energy models. It is important to note that the large scale Friedmann and small scale Newtonian dynamics implied by the modifications differ. They do so for the same reason that dark energy cannot be considered smooth relative to the dark matter on horizon scales and above: conservation of energy- 
momentum. The parameterization given in [10 spans both regimes and allows linear theory tools for general relativity to be used for modified gravity [33.

There does not yet exist a complete parameterization of the non-linear phenomenology of modified gravity but any viable description must contain a mechanism to suppress the modification locally. Density based approaches work well for the DGP model but less well for the potential driven $f(R)$ model. More importantly, the DGP and $f(R)$ models provide specific examples of this suppression. In both cases, the modification is mediated by a scalar that obeys a non-linear field equation coupled to density fluctuations that can be solved numerically via relaxation techniques [40. Non-linearities in the field equation prevent superposition of field solutions given the matter. In particular they allow saturation effects where the modifications are suppressed within the high overdensities where local tests of gravity are performed.

The drawback of the lack of superposition is that for cosmological tests, the field equations must be solved jointly with the matter in $N$-body simulations. In principle this caveat applies even to large scales in the supposedly linear regime. The DGP and $f(R)$ models however provide examples where the deviations from linear theory predictions there are small.

Likewise, the motion of matter within bound objects, while still responding gravitationally only to the metric, cannot always be thought of as a superposition of external and internal influences. These features caution against the use of simple scalings that take the linear behavior of modified gravity and seek to predict non-linear cosmological observables.

\section{REFERENCES}

1. A. J. Albrecht, et al. arXiv:astro-ph/0609591

2. A. J. Albrecht, et al. arXiv:0901.0721,

3. J. Frieman, M. Turner, D. Huterer, Ann. Rev. Astron. Astrophys. 46 (2008) 385-432, arXiv:0803.0982.

4. E. V. Linder, Rept. Prog. Phys. 71 (2008) 056901, arXiv:0801.2968.

5. S. M. Carroll, V. Duvvuri, M. Trodden, M. S. Turner, Phys. Rev. D70 (2004) 043528, arXiv:astro-ph/0306438

6. S. Nojiri, S. D. Odintsov, Phys. Rev. D68 (2003) 123512, arXiv:hep-th/0307288.

7. S. Capozziello, S. Carloni, A. Troisi, Recent Res. Dev. Astron. Astrophys. 1 (2003) 625, arXiv:astro-ph/0303041.

8. G. R. Dvali, G. Gabadadze, M. Porrati, Phys. Lett. B485 (2000) 208-214, arXiv:hep-th/0005016.

9. M. Kunz, D. Sapone, Phys. Rev. Lett. 98 (2007) 121301, arXiv:astro-ph/0612452.
10. W. Hu, I. Sawicki, Phys. Rev. D76 (2007) 104043, arXiv:0708.1190

11. W. Hu, Astrophys. J. 506 (1998) 485-494, arXiv:astro-ph/9801234

12. E. V. Linder, R. N. Cahn, Astropart. Phys. 28 (2007) 481, arXiv:astro-ph/0701317.

13. D. Huterer, E. V. Linder, Phys. Rev. D75 (2007) 023519, arXiv:astro-ph/0608681.

14. M. Ishak, A. Upadhye, D. N. Spergel, Phys. Rev. D74 (2006) 043513, arXiv:astro-ph/0507184.

15. S. Wang, L. Hui, M. May, Z. Haiman, Phys. Rev. D76 (2007) 063503, arXiv:0705.0165.

16. M. J. Mortonson, W. Hu, D. Huterer, Phys. Rev. D79 (2009) 023004, arXiv:0810.1744

17. U. Alam, V. Sahni, A. A. Starobinsky arXiv:0812.2846,

18. A. P. Hearin, A. R. Zentner arXiv:0904.3334.

19. Y. Song, W. Hu, I. Sawicki, Phys. Rev. D75 (2007) 044004, arXiv:astro-ph/0610532

20. I. Sawicki, Y. Song, W. Hu, Phys. Rev. D75 (2006) 064002, arXiv:astro-ph/0606285.

21. A. Cardoso, K. Koyama, S. S. Seahra, F. P. Silva, Phys. Rev. D77 (2008) 083512, arXiv:0711.2563.

22. L. Amendola, M. Kunz, D. Sapone, JCAP 0804 (2008) 013, arXiv:0704.2421.

23. J.-P. Uzan, Gen. Rel. Grav. 39 (2007) 307-342, arXiv:astro-ph/0605313.

24. R. Caldwell, A. Cooray, A. Melchiorri, Phys. Rev. D76 (2007) 023507, arXiv:astro-ph/0703375

25. W. Hu, D. J. Eisenstein, Phys. Rev. D59 (1999) 083509, arXiv:astro-ph/9809368.

26. E. Bertschinger, Astrophys. J. 648 (2006) 797-806, arXiv:astro-ph/0604485

27. I. Sawicki, Y.-S. Song, W. Hu, Phys. Rev. D75 (2007) 064002, arXiv:astro-ph/0606285.

28. Y. Song, I. Sawicki, W. Hu, Phys. Rev. D75 (2006) 064003, arXiv:astro-ph/0606286.

29. W. Fang, et al., Phys. Rev. D78 (2008) 103509, arXiv:0808.2208,

30. S. F. Daniel, R. R. Caldwell, A. Cooray, A. Melchiorri, Phys. Rev. D77 (2008) 103513, arXiv:0802.1068,

31. B. Jain, P. Zhang, Phys. Rev. D78 (2008) 063503, arXiv:0709.2375.

32. M. A. Amin, R. V. Wagoner, R. D. Blandford arXiv:0708.1793.

33. W. Hu, Phys. Rev. D77 (2008) 103524 , arXiv:0801.2433

34. W. Fang, W. Hu, A. Lewis, Phys. Rev. D78 (2008) 087303, arXiv:0808.3125.

35. L. Lombriser, W. Hu, W. Fang, U. Seljak arXiv:0905.1112

36. N. Afshordi, G. Geshnizjani, J. Khoury arXiv:0812.2244 
37. K. Koyama, A. Taruya, T. Hiramatsu arXiv:0902.0618.

38. D. Rapetti, S. W. Allen, A. Mantz, H. Ebeling arXiv:0812.2259.

39. J. Khoury, A. Weltman, Phys. Rev. D69 (2004) 044026, arXiv:astro-ph/0309411.

40. H. Oyaizu, Phys. Rev. D78 (2008) 123523, arXiv:0807.2449,

41. H. Oyaizu, M. Lima, W. Hu, Phys. Rev. D78 (2008) 123524, arXiv:0807.2462.

42. F. Schmidt arXiv:0905.0858.

43. F. Schmidt, M. V. Lima, H. Oyaizu, W. Hu arXiv:0812.0545.

44. L. Hui, A. Nicolis, C. Stubbs arXiv:0905.2966,

45. J. Khoury, M. Wyman arXiv:0903.1292.

46. A. Lue, R. Scoccimarro, G. D. Starkman, Phys. Rev. D69 (2004) 124015, arXiv:astro-ph/0401515.

47. K. Koyama, F. P. Silva, Phys. Rev. D75 (2007) 084040, arXiv:hep-th/0702169

48. A. Nicolis, R. Rattazzi, E. Trincherini arXiv:0811.2197. 